\title{
Investigation of the prevalence of LRV1 virus in clinical isolates of Leishmania (Viannia) spp. from patients with tegumentary leishmaniasis of a reference hospital
}

\section{Cristiele Saborito*, Elizabeth M. Coser, Edite H. Y. Kanashiro, José A. L. Lindoso, Adriano C. Coelho}

\begin{abstract}
Tegumentary leishmaniasis is a disease caused by a protozoan parasite belonging to the genus Leishmania. This disease can occur by four different forms: localized cutaneous, disseminated cutaneous, mucocutaneous or diffuse cutaneous. In Brazil, tegumentary leishmaniasis is caused mainly by the following species of the parasite: Leishmania (Viannia) braziliensis, L. (V.) guyanensis and L. (Leishmania) amazonensis, being the former one the most prevalent species. The virus LRV1 are double-stranded RNA virus found in species of Viannia subgenus, mainly L. (V.) braziliensis and L. (V.) guyanensis. Recent studies, in endemic areas in South America, have shown that there is a correlation between clinical treatment failure and the presence of Leishmania LRV1 virus in clinical isolates from patients. In this project, our objective is to evaluate the presence of LRV1 virus in 11 isolates of patients with tegumentary leishmaniasis of Instituto de Infectologia Emílio Ribas, São Paulo, SP.
\end{abstract}

\section{Key words:}

Leishmaniasis, Leishmania (Viannia) spp., LRV1 virus

\section{Introduction}

Tegumentary leishmaniasis is a disease caused by a protozoan of the genus Leishmania that can cause four clinical forms: localized cutaneous, disseminated cutaneous, mucocutaneous or diffuse cutaneous. The LRV1 is a double-stranded RNA virus that can be found in parasite species from Viannia subgenus. In Brazil, the main species in this subgenus are: $L$. (V.) braziliensis and $L$. (V.) guyanensis. Recent studies have shown that there is a correlation between clinical treatment failure and the presence of Leishmania LRV1 virus in clinical isolates of patients. The virus can favor the parasite persistence, increase evasion and metastasis, besides to active more pro-inflammatory cytokines. So this virus can affect the immune response from the host and the evolution of disease ${ }^{1}$. In this study, we propose to investigate the presence of LRV1 virus in Brazilian clinical isolates of subgenus Viannia, from patients with tegumentary leishmaniasis of Instituto de Infectologia Emilílio Ribas. There will be evaluated 11 clinical isolates from patients with tegumentary leishmaniasis that were previously typed as belonging the Viannia subgenus. The results will allow to evaluate if the therapeutic failures in treatment of these patients are correlated with the LRV1 virus.

\section{Results and Discussion}

Eleven clinical isolates from patients of Instituto de Infectologia Emílio Ribas were previously identified as belonging to the Viannia subgenus. Nine of these isolates are $L$. (V.) braziliensis, one is $L(V$.) guyanensis and the other one is $L$. (V.) shawi. For identification of the LRV1 virus, a RT-PCR assay was done using primers directed to the gene that codifies viral capsid and amplify a PCR product of approximately $680 \mathrm{bp}$. As positive control of reaction, it was used total RNA of the reference strain $L$. (V.) guyanensis (M4147). Until now, it was analyzed four clinical isolates of Leishmania, being identified the presence LRV1 virus in only one of these isolates (ER192) (Fig. 1). In the other isolates, the LRV1 virus was not detected by RT-PCR.

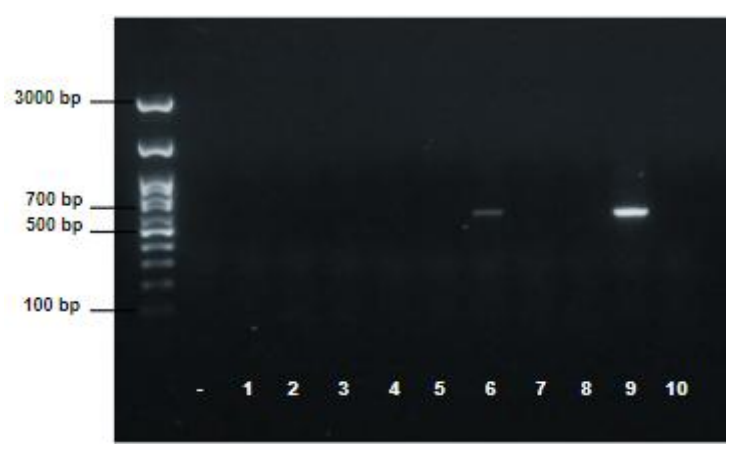

Figure 1. LRV1 virus identification in clinical isolates of $L$. (Viannia) spp. and in the reference strain $L$. (V.) guyanensis M4147, positive for the LRV1 virus. For the assays, the total RNA was purified from isolates and submitted to RT-PCR as previously described ${ }^{1}$. The samples were analyzed by agarose gel electrophoresis, stained with ethidium bromide. The samples 1 to 5 were done in the absence of the enzyme ReverseTranscriptase (RT), while the samples 6 to 10 in the presence of RT. Samples: 1 and 6 - L. (V.) guyanensis (M4147); 2 and 7 - L. (V.) braziliensis (ER010); 3 and 8 L. (V.) braziliensis (ER135); 4 and 9 - L. (V.) shawi (ER192); 5 and 10 - L. (V.) braziliensis (ER215).

\section{Conclusions}

The results obtained will provide data about the presence or not of LRV1 virus in these clinical isolates, allowing analyze if there is a correlation in patient's therapeutic failure and the LRV1 virus presence in these isolates.

\section{Acknowledgement}

This project is funded by Fundação de Amparo à Pesquisa do Estado de São Paulo (FAPESP 2016/211716). Cristiele Saborito and Elizabeth M. Coser are supported by FAPESP fellowships (FAPESP 2019/027176 and 2018/03299-0 respectively).

1 Ives et al., Science. 2011, 331(3018).

${ }^{2}$ Lye et al., PLoS Pathog. 2010, 6(10): e1001161 